\title{
Evaluación de proyectos de telecomunicaciones en la industria petrolera
}

\section{Model for the evaluation of telecom projects in the oil industry}

\author{
IDenys García de Mujica \\ garciadenys22@gmail.com \\ https://orcid.org/0000-0002-6690-8279 \\ Universidad Dr. Rafael Belloso Chacín, \\ Maracaibo-Venezuela
}

\author{
Nelson Bautista \\ bautistan@pdvsa.com \\ https://orcid.org/0000-0002-1335-3118 \\ PDVSA, Maracaibo-Venezuela
}

\author{
Adryd Mujica \\ mujica_adryd@hotmail.com \\ https://orcid.org/0000-0002-6326-5482 \\ ROSSETI, Córdoba-Argentina
}

RESUMEN El objetivo de este estudio fue diseñar un modelo para la evaluación de proyectos de telecomunicaciones en la industria petrolera. La investigación fue descriptiva y de campo, bajo la modalidad de proyecto factible, con diseño no experimental - transeccional. Se seleccionó una muestra de 22 sujetos al que se aplicó un cuestionario. La confiabilidad del instrumento se determinó mediante el coeficiente Alfa de Cronbach y arrojó un valor igual a 0,99. Los resultados con respecto a la variable "modelo para la evaluación de proyectos" reflejan una media aritmética correspondiente a 3,76 que se ubica en la categoría de alta manifestación y arroja un porcentaje igual a 32,59 \% para el nivel de respuesta "casi siempre". Se presentó una propuesta basada en cuatro fases: (a) ex-ante, (b) en proceso, (c) ex-post y (d) de impacto. Se evidenció la necesidad de establecer programas de actualización permanente sobre las tecnologías de telecomunicaciones, así como del proceso de evaluación de proyectos para coadyuvar a solventar las contingencias.

Palabras clave: Evaluación; Fases; Modelo; Proyecto; Telecomunicaciones

ABSTRACT The objective of this study is to design a model for the evaluation of telecommunications projects in the oil industry. The research was descriptive and field, under the modality of a feasible project. The design was non-experimental - transectional. A sample of 22 subjects was selected and a questionnaire was applied. The reliability of the instrument was determined using the Cronbach's alpha coefficient and it yielded a value equal to 0.99. The results with respect to the variable "Model for project evaluation" reflect an arithmetic mean corresponding to 3.76 which is in the category of high manifestation and yields a percentage equal to $32.59 \%$ for the response level "Almost always". A proposal based on four phases is presented: (a) Ex-ante, (b) In process, (c) Ex-post and (d) Impact. There was evidence of the need to establish, in the industry in the western part of the country, permanent updating programs on telecommunication technologies, as well as the process of evaluating projects to help solve contingencies.

Keywords: Evaluation; Phases; Model; Project; Telecommunications 


\section{INTRODUCCIÓN}

La industria petrolera diseña planes de desarrollo tecnológico con el propósito de determinar el nivel de obsolescencia de los equipos, adiciones y mejoras que se puedan presentar con el transcurrir del tiempo, para adaptarse a la demanda de solicitud de información, por lo tanto, es importante evaluar las tecnologías existentes en el mercado para realizar la selección más apropiada, según las necesidades existentes en la organización.

Lo antes expuesto, incentiva dentro de las organizaciones, la implantación y desarrollo de planes en proyectos de telecomunicaciones, para los cuales es necesaria la previa identificación de brechas, así como evitar dudas frente a situaciones, tales como: la cuantiosa inversión que esto representa, los niveles de riesgo asociados en cuanto a rentabilidad futura, entre otras.

Esta situación conlleva a la necesidad de fortalecerlos proyectosenmarcados en ladisciplina de telecomunicaciones, dentro de la industria petrolera. Se hace necesario que el ejecutivo de la industria petrolera otorgue la importancia requerida a la implantación y desarrollo de planes maestros de telecomunicaciones, los cuales actualmente no son considerados prioritarios debido a su escasa asociación con la generación de crudo.

En este orden de ideas, Homs (2003) señala que los sistemas de información y telecomunicaciones son esenciales para mejorar la productividad de las empresas, aunque su aplicación debe llevarse a cabo de forma inteligente; por tanto, para la implantación de nuevas tecnologías que produzcan rentabilidad, se necesita tener conocimiento profundo de los procesos de la empresa, a fin de planificar detalladamente las necesidades de tecnología de la información e incorporar los sistemas de información paulatinamente, empezando por los más elementales.

En función de lo planteado, para el proceso de evaluación de los proyectos de telecomunicaciones la organización debe contar con un modelo que proporcione resultados muy cerrados para evitar sus desviaciones, durante la ejecución de este.
Es relevante hacer mención, que cuando no se ejecutan proyectos que actualicen la plataforma de telecomunicaciones, la empresa comienza a poner en riesgo la productividad, los tiempos de entrega y la toma de decisiones a causa de retrasos, ya que no cuenta con la información a tiempo, lo cual se refleja en la producción con el aumento de movilización del personal de campo hacia las instalaciones, generándose un incremento del número de horas hombre laborales.

Por lo antes planteado, esta investigación tuvo como objetivo diseñar un modelo para la evaluación de proyectos de telecomunicaciones en la industria petrolera que permita dilucidar, cuando la implementación de estos proyectos es oportuna a pesar de las altas inversiones y riesgos asociados; $y$ al mismo tiempo, garantizar la viabilidad futura, en cuanto a aumentar los niveles de producción potencial y/o disminuir los costos de operación, lo cual a su vez genera ventajas para la empresa frente a los países pertenecientes a la Organización de Países Exportadores de Petróleo (OPEP).

\section{Fundamentación teórica}

Basado en los criterios de Crosby (1998), un modelo es una conceptualización de un evento, un proyecto, una hipótesis, el estado de una cuestión, que se representa como un esquema con símbolos descriptivos de características y relaciones más importantes con un fin, ser sometido a modelización como un diseño flexible, que emerge y se desarrolla durante el inicio de la investigación como una evaluación de su relevancia.

Por su parte, David (2013) señala que el modelo constituye un conjunto de objetos y sus relaciones, el cual describe la realidad; en particular, si permite hacer pronósticos o predicciones y, la emulación, representación en miniatura.

De acuerdo con lo expuesto por los autores, para efectos de esta investigación, se define modelo como una representación de la realidad, es decir, un conjunto de variables relacionadas entre sí e interactuantes, que en bloque dinámico conducen a obtener un resultado predeterminado para solucionar un problema. 
De acuerdo con los planteamientos de Sapag (2014), se define evaluación como el proceso que tiene como finalidad determinar el grado de eficacia y eficiencia, con que han sido empleados los recursos destinados a alcanzar los objetivos previstos, lo cual posibilita determinar las desviaciones y consecuencialmente la adopción de medidas correctivas que garanticen el cumplimiento adecuado de las metas presupuestadas.

En efecto, la evaluación se aplica ex ante (antes de), concomitante (durante), y ex post (después) de las actividades desarrolladas. Es importante hacer mención que la evaluación permite valorar cuantitativa y cualitativamente los resultados de la ejecución en las fases del proceso administrativo, haciendo posible medir en forma permanente el avance y los resultados de los proyectos, a fin de prevenir desviaciones y aplicar correctivos cuando sea necesario, con el objeto de retroalimentar la planificación.

Según Cartay (2010), un proyecto es un conjunto ordenado de acciones enfocado a un determinado fin $y$, por consiguiente, el mismo lleva consigo un inicio, alcanzándose su fin cuando todas sus actividades han sido completadas. De conformidad con la presente investigación, se entiende por proyecto la búsqueda, entre muchas, de una solución inteligente a una necesidad o problema a resolver; una necesidad humana, que, con sus diferencias, se satisface bajo una metodología definida, en función de la naturaleza de esta.

\section{Proceso para la evaluación}

El PMBOK (2017), expone que el proceso de evaluación es un conjunto de acciones y actividades interrelacionadas que se llevan a cabo para alcanzar un conjunto previamente especificado de productos, resultados o servicios. Es el equipo del proyecto, quien está a cargo de ejecutar los procesos de evaluación, que por lo general pertenecen a una de estas dos categorías principales: procesos de la dirección de proyectos y/o procesos orientados al producto. Los procesos para evaluar los proyectos abarcan todo el ciclo de vida del proyecto, el cual comprende: Iniciación, Planificación, Ejecución, Seguimiento y Control, y Cierre del proyecto; cumpliendo el ciclo que se muestra figura 1. Las mismas tienen dependencias claras y se llevan a cabo con la misma secuencia en cada proyecto.

Figura 1. El Ciclo Planificar-Hacer-Revisar-Actuar.

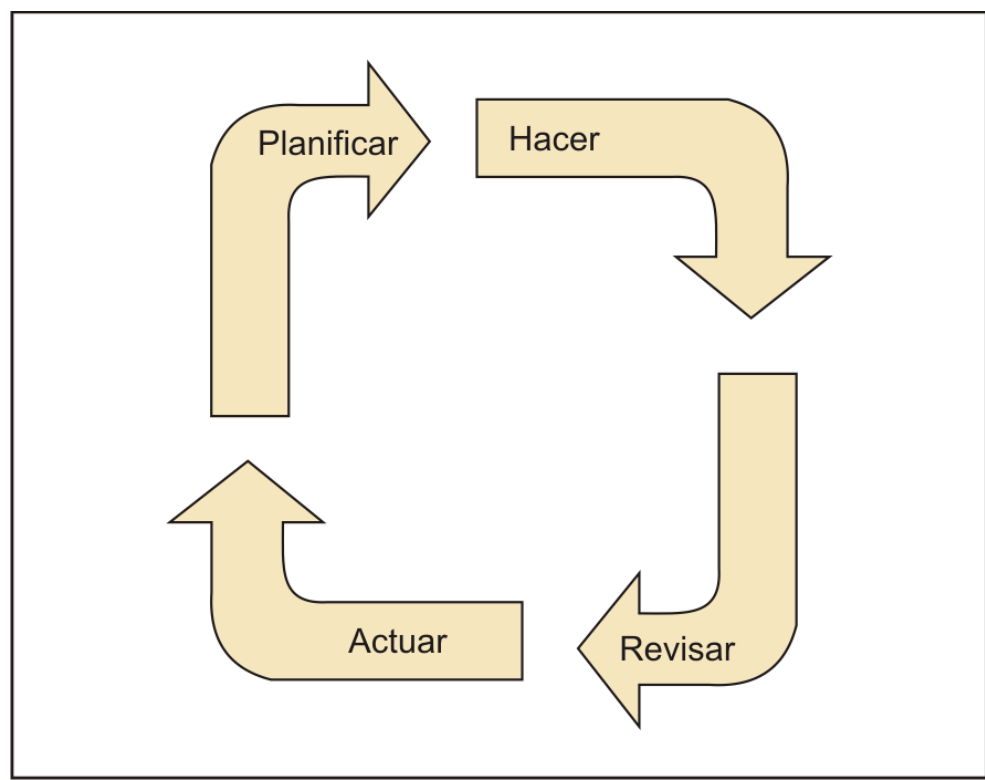

Fuente: PMBOK (2017) 
La figura 2, muestra como los procesos de seguimiento y control tienen incidencia en los demás procesos del proyecto.

Figura 2. Correspondencia de los Grupos de Procesos de Evaluación de Proyectos al ciclo Planificar-Hacer-Revisar-Actuar.

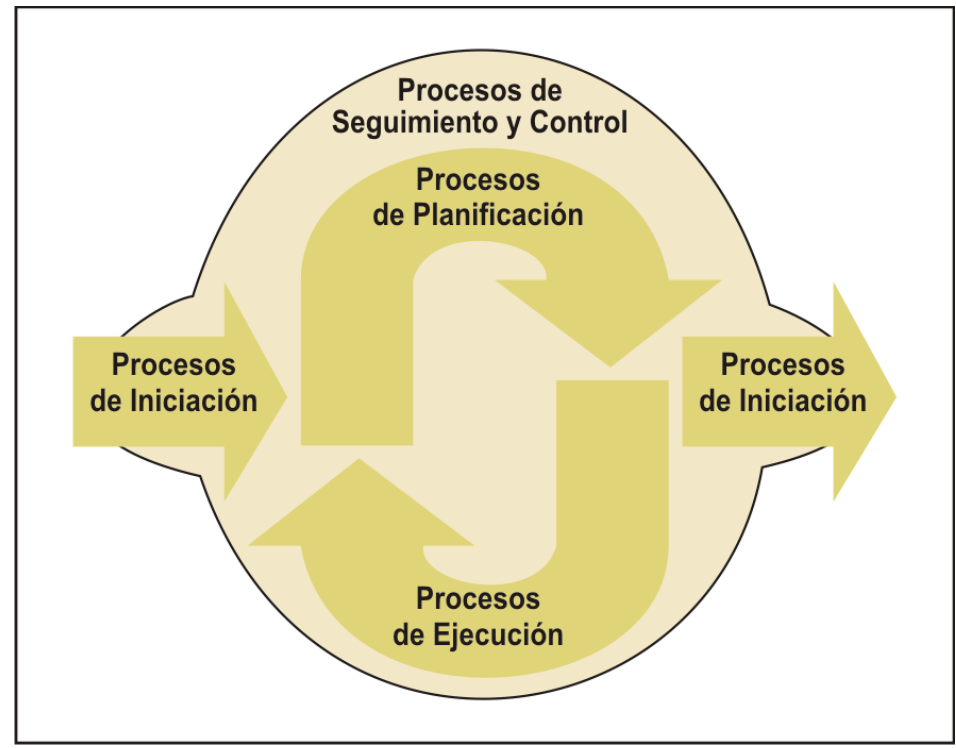

Fuente: PMBOK (2017)

Es válido hacer mención que, el proceso de planificación evalúa la definición, refina los objetivos y planifica el curso de acción requerido para lograr los objetivos y el alcance pretendido del proyecto. El proceso de ejecución: evalúa la integración de personas y otros recursos para llevar a cabo el plan de gestión del proyecto.

De igual forma, el proceso de seguimiento y control mide y supervisa regularmente el avance, a fin de identificar las variaciones respecto al plan de gestión del proyecto, de tal forma que se tomen medidas correctivas cuando sea necesario para cumplir con los objetivos del proyecto; en este proceso se practica una evaluación constante. Finalmente, el proceso de cierre formaliza la aceptación del producto, servicio o resultado, y termina ordenadamente el proyecto o una fase de este, y evalúa cada uno de los elementos.

En resumen, se puede decir que, los procesos de un proyecto enmarcan un conjunto de actividades que conllevan a que los proyectos en alguna medida lleguen a feliz término, para esto es recomendable seguir el ciclo de planificar, hacer, revisar y actuar; que permite mitigar la desviación de un proyecto, teniendo en cuenta siempre que un proyecto puede ser afectado por variables externas, las cuales no pueden ser controladas, por este proceso.

\section{Fases de los proyectos}

Antes de iniciar cualquier proceso para desarrollar un proyecto, es necesario tener claro cuáles de las fases de un proyecto, que de acuerdo con la Guía de Gerencia para Inversión Capital - GGIPC (PDVSA, 1999) y Cartay (2010) son las siguientes: visualización, conceptualización, definición, implantación y operación. Las fases se encuentran claramente delimitadas y evaluadas a través de los documentos de soporte de decisión (DSD), los cuales sustentan la viabilidad de cada una de estas, contienen los resultados de la correspondiente evaluación e indican el cumplimiento de los objetivos establecidos para la misma; si el documento de soporte de decisión no tiene los recaudos necesarios, el proyecto se replantea o reestructura para ajustarse a la realidad de los recursos. Estos documentos se encuentran identificados en la figura 3 con los indicadores D1, D2, D3 y D4. 
Figura 3. Proceso de ejecución de los proyectos.

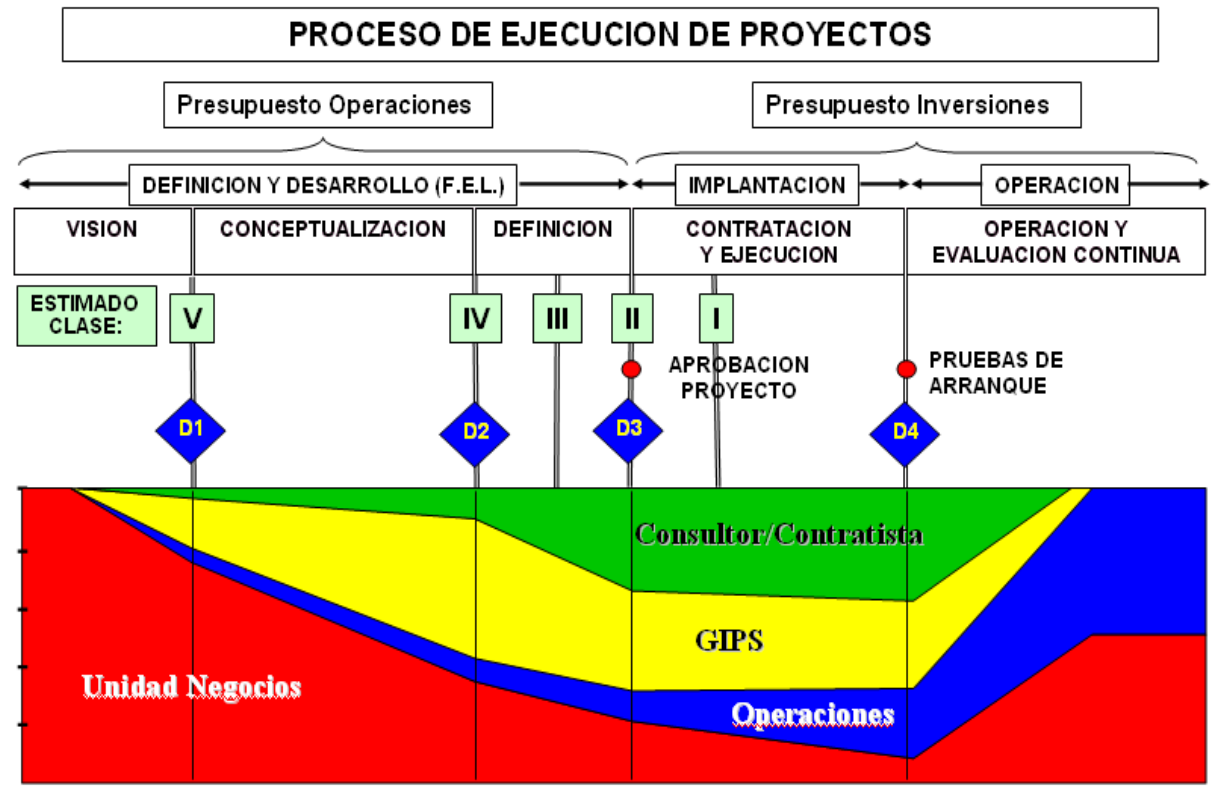

Fuente: PDVSA (1999)

En la primera fase, denominada visualización, se originan los proyectos de inversión. Según la GGPIC (PDVSA, 1999), las ideas que originan los proyectos pueden provenir, en cualquier momento, de cualquier parte de la corporación, pero son generalmente el producto de los análisis del ambiente externo e interno a ella, o del análisis F.O.D.A (Fortalezas, Oportunidades, Debilidades, Amenazas) que se realiza como parte de los ciclos de planificación. Estos análisis se efectúan en equipo con la participación de todas las organizaciones involucradas en el proyecto y bajo la responsabilidad integradora de las unidades de planificación corporativa.

La fase siguiente o fase de conceptualización, es la fase en la cual se procesan los productos resultantes de la fase de visualizar, que vienen a ser el insumo de trabajo para continuar con el desarrollo del proyecto mediante la conceptualización, cuyo objetivo es la selección de la(s) mejor(es) opción(es) y la mejora en la precisión de los estimados de costos y tiempo de implantación. Todo esto con el propósito de lograr la reducción de la incertidumbre y cuantificar los riesgos asociados, así como, determinar el valor esperado para la(s) opción(es) seleccionada(s).

Básicamente, la fase de conceptualización busca cumplir con dos aspectos como son: la definición, donde las decisiones tomadas en la fase de conceptualización constituyen el insumo de trabajo para continuar con el desarrollo del proyecto mediante la ejecución de las correspondientes definiciones. Según la GGPIC (PDVSA, 1999), el propósito de esta fase es desarrollar en detalle el alcance y los planes de ejecución de la opción seleccionada para permitir a la corporación comprometer los fondos u obtener el financiamiento requerido para ejecutar el proyecto; del mismo modo, preparar la documentación que sirva de base para la ingeniería de detalle; la contratación de la ejecución del proyecto y; confirmar si el valor esperado del proyecto cumple con los objetivos del negocio.

Definir consiste en llevar a cabo tres procesos esencialmente básicos: en primera instancia, 
desarrollar el paquete de definición del proyecto, para lo cual es necesario ejecutar las siguientes actividades, analizar los riesgos, precisar el alcance y elaboración del diseño básico; asimismo, desarrollar en detalle el plan de ejecución, como también, la preparación del estimado de costos Clase II, la evaluación del grado de definición del proyecto; así como, el establecimiento de las guías para el control del proyecto y desarrollar el plan de aseguramiento tecnológico.

Como segunda instancia, es el establecimiento del proceso de contratación y el documento de solicitud de ofertas (D.S.O), el cual consiste en la planificación y ejecución de todas las actividades que conllevan a un entendimiento formal entre el dueño del proyecto y uno o más contratistas. Este entendimiento llamado contrato, tiene carácter legal, ya que los compromisos que se acuerdan entre las partes pasan a ser de obligatorio cumplimiento ante la ley. Un contrato formal otorga ventajas al facilitar el desarrollo de términos y condiciones aceptables por ambas partes.

Es importante hacer mención, que las actividades de contratación per sé están comprendidas en dos diferentes fases de la vida del proyecto (Definición e Implantación) y no siempre con la misma distribución (cada proyecto es un caso único). En la figura 4 se observa cómo se mueve el desembolso en un proyecto.

Figura 4. Proceso de ejecución de los proyectos.

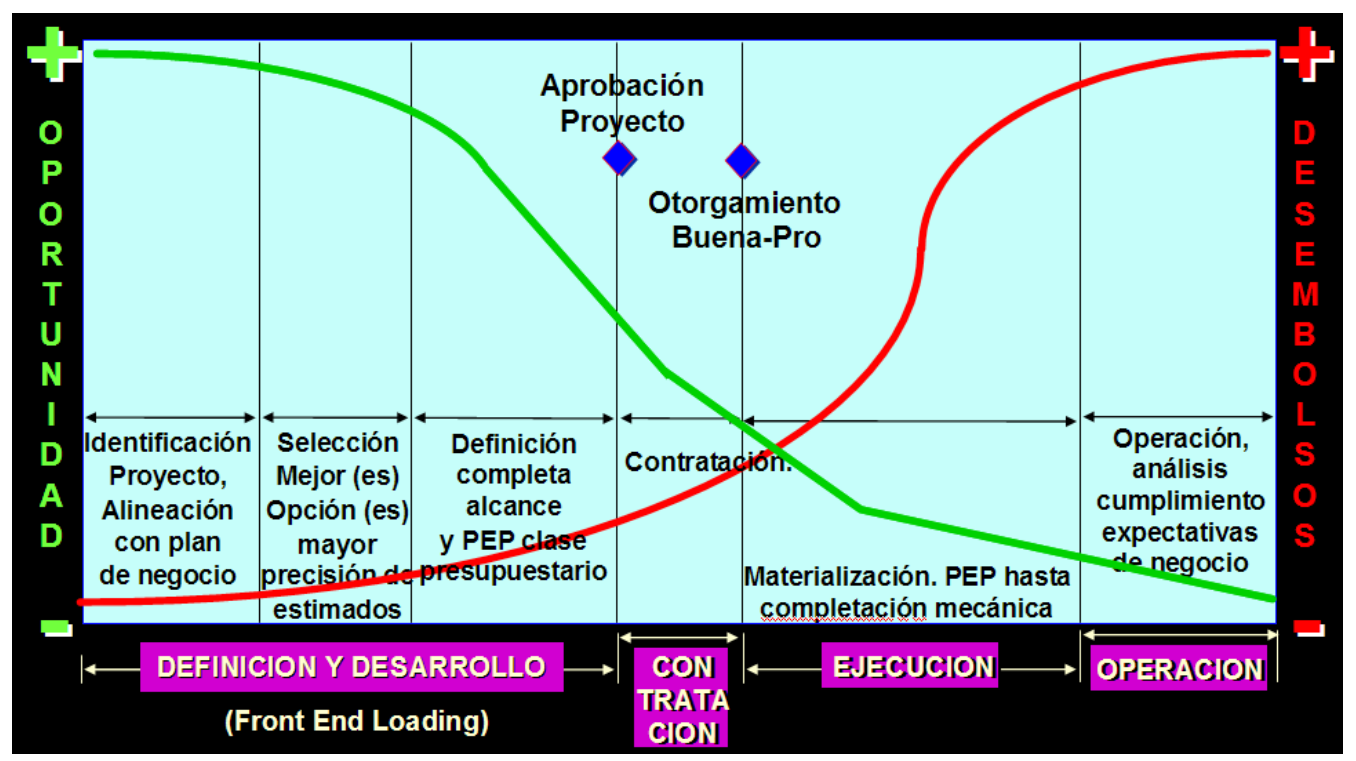

Fuente: PDVSA (1999)

Desde luego, una vez que se está claro con la ingeniería, se procede con la procura de materiales, actividad primordial para lograr la ejecución (construcción) del proyecto dentro del tiempo ycon la calidad planificada. Esto se logra, básicamente, mediante la coordinación de programas de seguimiento, control y aseguramiento de calidad de los materiales. Para finalizar esta fase se requiere contar con la gerencia de construcción en donde el dueño debe velar para que los siguientes aspectos del proyecto sean discutidos y acordados con el contratista, una vez otorgado el contrato de construcción es necesario discutir: facilidades de campo, supervisión de la construcción, materiales y equipos, relaciones laborales, apoyo al arranque y apoyo operacional. 
En la práctica, es difícil establecer el momento específico cuando termina la construcción y comienza la fase de operación, ya que existe un período de solapamiento, generalmente al finalizar la construcción mecánica. Durante este período, el rol de coordinador pasa del gerente de construcción al de arranque, quien será a partir de ese momento el líder responsable por alcanzar el cierre del proyecto.

En la fase de operación es cuando se evalúa la operación de lo construido, se realizan las pruebas de garantía en donde se verifica que todo está construido acorde a lo especificado por el fabricante, con el fin de no perder la garantía. En esta fase también se involucra al cliente, quien finalmente va a aceptar y recibir la construcción - la instalación, en el caso de proyectos de telecomunicaciones para la industria petrolera, como es el caso de esta investigación, el cliente es la gerencia de telecomunicaciones.

En la evaluación de proyectos de tecnologías de información y comunicación el problema que se presenta al intentar evaluarlo es el decidir cómo manejar la incertidumbre que estos poseen. Según Pérez (2002), las evaluaciones se basan en estimaciones de lo que se espera sean en el futuro los beneficios y costos que se asocian a un proyecto. Así mismo, las evaluaciones también se basan en una serie de decisiones que deben ser tomadas por quién realiza la evaluación, por lo que es necesario contar con profesionales con conocimientos y experiencia en el campo de aplicación a fin de obtener resultados adecuados a los requerimientos del cliente.

Es natural pensar que los proyectos, y en particular los de telecomunicaciones, posean un alto riesgo asociado. Por lo que es recomendable prestar especial atención y tener cuidado al momento de incorporar los riesgos presentes dentro de la evaluación económica del proyecto.

Según los enunciados de Remeyi, Money, Sherwood-Smith e Irani (2000), es importante realizar un análisis detallado y cuidadoso de las cualidades y características que incorpora una inversión en Tecnología de Información (TI) y Sistemas de Información (SI), por razones como: usualmente involucran grandes sumas de dinero, no siempre son percibidas como ganancias directas o fuentes de ganancias para el negocio, no todas las veces hay acuerdo sobre la necesidad de invertir en esa área, muchos de los gastos en telecomunicaciones, especialmente en hardware, tradicionalmente se han capitalizado, han tenido un crecimiento poco satisfactorio en el desarrollo de las funciones de telecomunicaciones y existe un alto riesgo asociado a los proyectos de inversión en telecomunicaciones, entre otras.

Por otra parte, es importante considerar la postura de Strassmann (1997), quién argumenta que el valor de las TIC's dentro de las organizaciones aún no ha dejado de ser un valor basado fundamentalmente en el mejoramiento operacional de la productividad, para convertirse en forma plena en un valor basado en contribución al negocio mismo.

La opinión del autor antes mencionado, veladamente recomienda que antes de esperar obtener mejoras en el proceso de evaluación de proyectos de telecomunicaciones, las organizaciones deben preocuparse en estar operacionalmente productivas y óptimo funcionamiento, de lo contrario, no será posible obtener ventajas comparativas que apoyen los objetivos estratégicos del negocio, y los ejecutivos de negocios continuarán desconfiando de las estimaciones y evaluaciones que realicen los ejecutivos de tecnologías de información con respecto a proyectos de inversión en telecomunicaciones.

De esta forma, los modelos de evaluación de proyectos para las áreas de tecnologías de información y comunicación se dividen principalmente según el nivel de gestión, de acuerdo a la naturaleza de la evaluación y en función del momento en que se realiza. 
Aramburú (2001) expresa que, los distintos modelos de evaluación varían según el momento en que se realicen, y considera que estos están compuestos por las fases siguientes: ex-ante, de proceso, ex-post y de impacto. De tal manera, que Pérez (2002), apoyándose en lo expuesto por Remeyi, Money, Sherwood-Smith, e Irani (2000) plantea que, entre las muchas propuestas y modelos de evaluación existentes, en forma general se encuentran dos grupos: evaluación Exante y Ex-post, y evaluación formativa. En la figura 5 se presenta gráficamente la evaluación en el ciclo del proyecto.

De esta manera, es entendida la evaluación exante, por el mismo autor, como la que consiste en seleccionar entre varias alternativas técnicamente factibles aquella que produce el mayor impacto al mínimo costo, se toman en cuenta todos los aspectos mencionados en el proceso de iniciación y la fase de visualización, esto se efectúa antes de la aprobación del proyecto y busca conocer su pertinencia, viabilidad y eficacia potencial; contempla todas las necesidades expuestas por el cliente. Según Medianero (1998) este tipo de evaluación supone la incorporación de ajustes necesarios en el diseño del proyecto, lo cual podría generar incluso el cambio del grupo beneficiario, su jerarquía de objetivos y el presupuesto. Entre otros postulados que apoyan esta línea, se tiene el de Pérez (2002) que, dentro de la fase considera las estimaciones y pronósticos financieros, de costos y beneficios; lo que contribuye a decidir si el proyecto en cuestión será o no realizado.

Figura 5. La evaluación en el ciclo de proyectos.

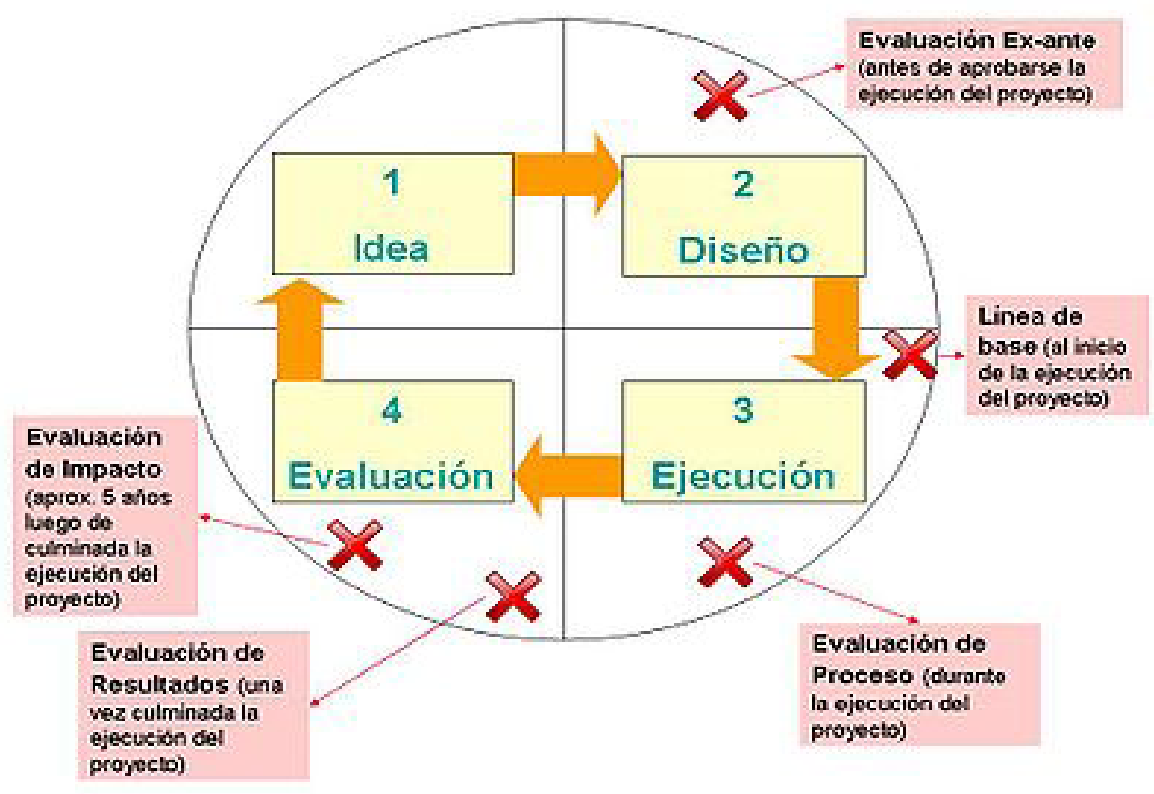

Fuente: Pérez (2002) 
En referencia a la evaluación de proceso operativa, de medio término o continua: esta se hace a medida que el proyecto va desarrollándose y guarda estrecha relación con su monitoreo. La misma, permite conocer en qué medida se está alcanzando el logro de los objetivos (Resultados en caso de marco lógico). Una evaluación de este tipo debe buscar aportar al perfeccionamiento del modelo de intervención empleado y a identificar lecciones aprendidas. Las fuentes financieras suelen requerir la realización de este tipo de evaluación para ejecutar los desembolsos periódicos.

De la misma forma, la evaluación ex-post, de resultados o de fin de proyecto, se realiza al culminar el proyecto. Esta se enfoca en indagar el nivel de cumplimiento de los objetivos (propósito y resultados en caso de marco lógico); asimismo, busca demostrar que los cambios producidos son consecuencia de las actividades del proyecto (exclusivamente o en interacción con otras fuentes); para esto suele recurrir a un diseño experimental. No solo indaga por cambios positivos, también analiza efectos negativos e inesperados. Pérez (2002) considera, que las evaluaciones ex-post se enfocan en temas críticos de la inversión, una vez realizada esta, la idea es confirmar o refutar el valor que alcanzó el desarrollo del proyecto. En este caso, se utiliza o se propone el análisis de las inversiones en tecnología de información en términos de indicador o de un conjunto de indicadores, tales como el VAN, la TIR y el EVA, entre otros.

Por su parte, Aramburú (2001) expresa que, la evaluación de impacto es la que indaga por los cambios permanentes y las mejoras de la calidad de vida producida por el proyecto, es decir, se enfoca en conocer la sostenibilidad de los cambios alcanzados y los efectos imprevistos (positivos o negativos). En caso de diseño con marco lógico, se enfoca en la evaluación del fin de la jerarquía de objetivos. Esta evaluación necesariamente debe ser realizada luego de un tiempo de culminado el proyecto y no de inmediato a su conclusión; el tiempo recomendado para efectuarla es de 5 años.

Según el Banco Interamericano de Desarrollo (1997), cabe considerar que las evaluaciones ex-ante y de proceso, son consideradas como evaluaciones formativas debido a que se producen mientras se da la preparación y/o ejecución del proyecto y sus conclusiones sirven para optimizar la ejecución del mismo; en tanto que, las evaluaciones de resultados y de impacto vienen a ser evaluaciones sumativas que ocurren al culminar el proyecto e incluso un tiempo después de haber culminado, por lo que sus conclusiones, aun cuando ya no podrán tener una aplicación directa en el proyecto que finalizó, sirven para ser transferidas a otras experiencias.

En este sentido, Hewett (1986) expresa que, al ser aplicada al desarrollo de sistema de comunicación o computacionales, la evaluación formativa involucra monitorear el proceso y los resultados del sistema de desarrollo, y retroalimentarse con las opiniones de los usuarios para ser utilizados en su refinamiento y en nuevos sistemas. La evaluación sumativa involucra entonces, analizar el impacto que se traduce en el uso de lo creado por el proyecto y la efectividad de este; así como el desempeño del sistema de comunicación.

\section{METODOLOGÍA}

En la investigación se manejó la variable modelo para la evaluación de proyectos de telecomunicaciones, definida operacionalmente como el proceso a través del cual se sistematizan los procedimientos relacionados con las fases de evaluación de proyectos, a fin de lograr, las metas propuestas al inicio de este, en el tiempo estipulado y bajo los costos presupuestados, para esto se establecieron los criterios de evaluación de la industria petrolera. 
La investigación fue de tipo proyecto factible, no experimental y con un diseño de campo, descriptivo transeccional. La población estuvo conformada por veintidós informantes claves, de las gerencias de telecomunicaciones de la industria petrolera, con mayor experiencia en el área de evaluación de proyectos de telecomunicaciones. La técnica de recolección de datos correspondió a la encuesta y el instrumento utilizado fue un cuestionario, este último validado mediante el juicio de expertos, con una confiabilidad de 0,99 que le otorga una muy alta confiabilidad. Los resultados fueron analizados mediante la estadística descriptiva.

Es importante señalar que, para el análisis estadístico de los resultados se diseñó un baremo de interpretación, basado en los criterios de Ruíz (2002), el cual contiene el rango, el intervalo y la categoría, para poder explicar el razonamiento de los resultados, el cual se muestra en la Tabla 1.

Tabla 1. Baremo para la categorización estadística de la variable.

\begin{tabular}{cccc}
\hline ALTERNATIVA & RANGO & CATEGORIA & SIGNIFICACIÓN \\
\hline Siempre & $4,20-5$ & MUY ALTA & Muy alta manifestación \\
Casi siempre & $3,40-4,19$ & ALTA & Alta manifestación \\
En forma moderada & $2,60-3,39$ & MEDIANA & Mediana manifestación \\
Casi nunca & $1,80-2,59$ & BAJA & Baja manifestación \\
Nunca & $1-1,79$ & MUY BAJA & Muy Baja manifestación \\
\hline
\end{tabular}

Fuente: adaptado de Ruíz (2002)

RESULTADOS

A continuación, se presentan los resultados de la investigación, obtenidos a través de la aplicación del instrumento de recolección datos, el cual se diseñó con las alternativas de respuesta: siempre (5), casi siempre (4), algunas veces (3), casi nunca (2) y nunca (1); así como también, el análisis de estos. Estos resultados sirvieron de base para dar respuesta a las interrogantes planteadas inicialmente en la investigación, conocer las desviaciones existentes en cuanto a la evaluación de proyectos se refiere, y lograr con base a los resultados, diseñar el modelo propuesto para la evaluación de proyectos de telecomunicaciones.

Los resultados se muestran tabulados en forma de frecuencias absolutas (fa), porcentajes (\%) y medias aritméticas ( ), siendo la una medida de tendencia central. Es importante destacar que, el análisis porcentual se efectuó agrupando los datos para cada dimensión que conforma la variable de estudio para su posterior análisis e interpretación.

A continuación, se presenta la Tabla 2 que muestra los resultados obtenidos con respecto a la dimensión proceso de evaluación de proyectos de telecomunicaciones. 
Tabla 2. Proceso de evaluación de proyectos.

\begin{tabular}{|c|c|c|c|c|c|c|c|c|c|c|c|c|}
\hline \multirow{2}{*}{ ALTERNATIVAS } & \multicolumn{2}{|c|}{ Siempre } & \multicolumn{2}{|c|}{$\begin{array}{c}\text { Casi } \\
\text { siempre }\end{array}$} & \multicolumn{2}{|c|}{$\begin{array}{l}\text { Algunas } \\
\text { veces }\end{array}$} & \multicolumn{2}{|c|}{$\begin{array}{c}\text { Casi } \\
\text { nunca }\end{array}$} & \multicolumn{2}{|c|}{ Nunca } & \multicolumn{2}{|c|}{ Total } \\
\hline & $F: A$ & $\%$ & F:A & $\%$ & F:A & $\%$ & F:A & $\%$ & F:A & $\%$ & $F: A$ & $\%$ \\
\hline $\begin{array}{l}\text { Procesos para la } \\
\text { evaluación }\end{array}$ & 105 & 37,88 & 105 & 31,82 & 63 & 19,09 & 16 & 4,85 & 21 & 6,36 & 330 & 100 \\
\hline$\overline{\boldsymbol{X}}$ Dimensión & & & & & & 3,94 & & & & & & \\
\hline
\end{tabular}

Los resultados de la Tabla 2, muestran una marcada inclinación positiva, donde la mayoría de los encuestados respondió siempre con un $37,88 \%$, seguido de casi siempre con $31,82 \%$, algunas veces con $19,09 \%$, nunca con $6,36 \%$ y, por último, la alternativa casi nunca con un $4,85 \%$.

Estos resultados indican que los sujetos de la muestra siempre están realizando algún tipo de procesos de evaluación, lo cual va acorde con lo expuesto en el PMBOK (2017), donde indica que un proceso es un conjunto de acciones y actividades interrelacionadas que se llevan a cabo para alcanzar un conjunto previamente especificado de productos, resultados o servicios. Sobre la base de los resultados, se observa que la tendencia central de la dimensión es de 3,94; lo cual se traduce en una alta manifestación de las actividades del proceso de evaluación de proyectos, por lo que se puede deducir que el equipo del proyecto es el que está a cargo de ejecutar los procesos de evaluación de proyectos.

Con relación a la segunda dimensión denominada ejecución de las fases de los proyectos, en la Tabla 3 se presentan los resultados obtenidos, a través de la aplicación del instrumento, para esta dimensión:

Tabla 3. Ejecución de las fases de los proyectos.

\begin{tabular}{|c|c|c|c|c|c|c|c|c|c|c|c|c|}
\hline \multirow{2}{*}{ ALTERNATIVAS } & \multicolumn{2}{|c|}{ Siempre } & \multicolumn{2}{|c|}{$\begin{array}{l}\text { Casi } \\
\text { siempre }\end{array}$} & \multicolumn{2}{|c|}{$\begin{array}{l}\text { Algunas } \\
\text { veces }\end{array}$} & \multicolumn{2}{|c|}{$\begin{array}{c}\text { Casi } \\
\text { nunca }\end{array}$} & \multicolumn{2}{|c|}{ Nunca } & \multicolumn{2}{|c|}{ Total } \\
\hline & $\mathrm{F}: \mathrm{A}$ & $\%$ & F:A & $\%$ & $F: A$ & $\%$ & F:A & $\%$ & $F: A$ & $\%$ & F:A & $\%$ \\
\hline $\begin{array}{c}\text { Ejecución de las fases } \\
\text { de los proyectos }\end{array}$ & 157 & 32,44 & 164 & 33,88 & 81 & 16,74 & 52 & 10,74 & 30 & 6,2 & 484 & 100 \\
\hline $\bar{X}$ Dimensión & & & & & & 3,79 & & & & & & \\
\hline
\end{tabular}

En la tabla 3 se indican los resultados obtenidos en la investigación al momento de ejecutar las fases de los proyectos, observándose que la mayoría de los sujetos respondió casi siempre con un $33,88 \%$, seguido de siempre con $32,44 \%$, y la alternativa algunas veces con $16,74 \%$, casi nunca con $10,74 \%$ y por último la alternativa nunca con un $6,20 \%$. De igual forma, arrojó un valor igual a 3,79 como tendencia central de la dimensión, lo que indica que existe una alta manifestación por parte del equipo de trabajo a aplicar las fases del proyecto, lo cual coincide con lo definido por la GGPIC (PDVSA, 1999).

En el proceso de ejecución de las fases de los proyectos, se encontró el más bajo resultado en la fase de contratación, donde prevaleció la ejecución de la contratación a mediano plazo; lo cual es desfavorable para proyectos sobre todo en los inherentes a telecomunicaciones, ya que según Homs (2003), los sistemas de información y 
telecomunicaciones son esenciales para mejorar la productividad de las empresas y su aplicación debe llevarse a cabo de forma inteligente, por lo tanto, para la implantación de nuevas tecnologías que produzcan rentabilidad, se necesita tener conocimiento profundo de los procesos de contratación en la empresa, de forma que se puedan planificar detalladamente las necesidades de tecnología de la información e incorporar los sistemas de información paulatinamente, empezando por los más elementales. Aparte de la desventaja mencionada, el resto de los indicadores reflejaron una respuesta favorable en el estudio.

Tabla 4. Requerimientos para la evaluación.

\begin{tabular}{|c|c|c|c|c|c|c|c|c|c|c|c|c|}
\hline \multirow{2}{*}{ ALTERNATIVAS } & \multicolumn{2}{|c|}{ Siempre } & \multicolumn{2}{|c|}{$\begin{array}{c}\text { Casi } \\
\text { siempre }\end{array}$} & \multicolumn{2}{|c|}{$\begin{array}{l}\text { Algunas } \\
\text { veces }\end{array}$} & \multicolumn{2}{|c|}{$\begin{array}{c}\text { Casi } \\
\text { nunca }\end{array}$} & \multicolumn{2}{|c|}{ Nunca } & \multicolumn{2}{|c|}{ Total } \\
\hline & $F: A$ & $\%$ & F:A & $\%$ & F:A & $\%$ & F:A & $\%$ & F:A & $\%$ & $F: A$ & $\%$ \\
\hline $\begin{array}{c}\text { Requerimientos para la } \\
\text { evaluación }\end{array}$ & 8,2 & 33,88 & 85 & 35,12 & 44 & 18,18 & 15 & 6,2 & 16 & 6,61 & 242 & 100 \\
\hline $\bar{X}$ Dimensión & & & & & & 3,86 & & & & & & \\
\hline
\end{tabular}

Los datos de la tabla 4, definen los resultados de la dimensión requerimientos para la evaluación de proyectos, los cuales arrojaron una marcada inclinación positiva, donde la mayoría de los sujetos de la muestra seleccionaron la alternativa casi siempre con $35,12 \%$; seguida de la opción siempre con $33,88 \%$ y algunas veces con $18,18 \%$; un 6,61 $\%$ para la opción nunca y $6,20 \%$ para casi nunca. De los resultados se deduce que los proyectos en la industria son desarrollados de acuerdo con los procesos, arrojando alta manifestación con una media de la dimensión de 3,86. Estos resultados coinciden con los planteamientos de Gido, Jacks y Clements (1999), quien expresa que, los requerimientos para la evaluación de un proyecto como son cliente, tiempo, presupuesto, recursos y organización deben ser considerados durante la ejecución del proyecto.

Tabla 5. Fases de los proyectos.

\begin{tabular}{|c|c|c|c|c|c|c|c|c|c|c|c|c|}
\hline \multirow{2}{*}{ ALTERNATIVAS } & \multicolumn{2}{|c|}{ Siempre } & \multicolumn{2}{|c|}{$\begin{array}{c}\text { Casi } \\
\text { siempre }\end{array}$} & \multicolumn{2}{|c|}{$\begin{array}{c}\text { Algunas } \\
\text { veces }\end{array}$} & \multicolumn{2}{|c|}{$\begin{array}{c}\text { Casi } \\
\text { nunca }\end{array}$} & \multicolumn{2}{|c|}{ Nunca } & \multicolumn{2}{|c|}{ Total } \\
\hline & $F: A$ & $\%$ & $F: A$ & $\%$ & F:A & $\%$ & F:A & $\%$ & F:A & $\%$ & F:A & $\%$ \\
\hline Fases de los proyectos & 157 & 32,44 & 164 & 33,88 & 81 & 16,74 & 52 & 10,74 & 30 & 6,2 & 484 & 100 \\
\hline $\bar{X}$ Dimensión & & & & & & 3,76 & & & & & & \\
\hline
\end{tabular}

La dimensión fases de los proyectos, Tabla 5 , arrojó una marcada inclinación positiva, donde la mayoría de la muestra respondió casi siempre con un $33,88 \%$, seguido de siempre con $32,44 \%$, y la alternativa algunas veces con $16,74 \%$, casi nunca con $10,74 \%$ y por último la alternativa nunca con un $6,20 \%$; dando como resultado una media de la dimensión de 3,76; indicando una alta manifestación en la ejecución de las fases de los proyectos, lo cual de alguna manera favorece la ejecución del proyecto y coincide con los planteamientos de la GGPIC (PDVSA, 1999), cuando indica la necesidad de aplicar las fases en la búsqueda del alcance de los objetivos establecidos para el proyecto. 
Tabla 6. Resultados de la variable.

\begin{tabular}{|c|c|c|c|c|c|c|c|c|c|c|c|c|}
\hline \multirow{2}{*}{ ALTERNATIVAS } & \multicolumn{2}{|c|}{ Siempre } & \multicolumn{2}{|c|}{$\begin{array}{c}\text { Casi } \\
\text { siempre }\end{array}$} & \multicolumn{2}{|c|}{$\begin{array}{c}\text { Algunas } \\
\text { veces }\end{array}$} & \multicolumn{2}{|c|}{$\begin{array}{c}\text { Casi } \\
\text { nunca }\end{array}$} & \multicolumn{2}{|c|}{ Nunca } & \multicolumn{2}{|c|}{ Total } \\
\hline & F:A & $\%$ & F:A & $\%$ & F:A & $\%$ & F:A & $\%$ & $F: A$ & $\%$ & F:A & $\%$ \\
\hline $\begin{array}{l}\text { Modelo para la } \\
\text { evaluación de } \\
\text { proyectos }\end{array}$ & 458 & 32,03 & 466 & 32,59 & 265 & 18,53 & 129 & 9,02 & 112 & 7,83 & 1430 & 100 \\
\hline $\bar{X}$ Dimensión & & & & & & 3,76 & & & & & & \\
\hline
\end{tabular}

En la tabla 6 se muestran los resultados obtenidos para la variable Modelo para la evaluación de proyectos, los cuales presentan una marcada inclinación positiva, donde la mayoría de los sujetos de la muestra seleccionaron la alternativa casi siempre con $32,59 \%$; seguida de la opción siempre con 32,03\% y algunas veces con 18,53\%; marcando en la zona negativa un $9,02 \%$ para la opción casi nunca y $7,83 \%$ para la alternativa nunca.

Los resultados, manejados de manera integral para la variable, indican que los proyectos son evaluados bajos los postulados de PMBOK (2017), Pérez (2002), Aramburú (2001), Gido y Clements, Jacks y Clements (1999) y GGPIC (PDVSA, 1999), tal como se visualiza en la tendencia central con un valor de 3,76; sin embargo, se encontraron algunas debilidades que son recomendables al considerar el modelo propuesto, tales como: manejo de la información, preparación del plan para conceptualizar el proyecto. Evaluación del grado de definición del proyecto, proceso de contratación en los aspectos selección de contratistas y evaluación continua, entre otros.

Finalmente, se infiere que los resultados obtenidos en las distintas dimensiones son elementos de relevancia que deben ser considerados en un modelo de evaluación de proyectos de automatizaciones, ya que están relacionados con el proceso y sus fases e involucran las características propias de los proyectos como son el tiempo, costo y la calidad de este. De igual manera, se presentan los puntos de atención considerados como generadores de desviaciones y retrasos para la culminación exitosa del proyecto dentro del tiempo establecido y con los recursos programados.

Asimismo, se dedujo del análisis que, existe entre los líderes y gerentes de los proyectos de telecomunicaciones amplio conocimiento de los procesos de evaluación, lo que converge en la necesidad de la aplicación de las fases del proyecto.

\section{Propuesta del modelo para la evaluación de proyectos en la industria petrolera}

En función de los resultados obtenidos en esta investigación, a través de los cuales se pudo evidenciar la necesidad de la industria petrolera en cuanto a disponer de una herramienta que le brinde una mejor forma de evaluar los proyectos del sector petrolero, se presenta a continuación una propuesta de un modelo para la evaluación de proyectos en el sector petrolero.

El modelo propuesto se define como un conjunto de procesos continuos, periódicos y sistematizados de recolección y análisis de la información para ser evaluados, que se lleva a cabo desde la iniciación hasta la operación de un proyecto, con la finalidad de conocer sus valores y aportes a la industria, identificar desviaciones y aplicar los correctivos necesarios para que este se desarrolle eficaz y eficientemente en su fase continua, a su vez; estos procesos se 
interrelacionan entre sí, brindando un conjunto de variables que facilitan la evaluación de un proyecto en la industria petrolera y su aplicación a los del sector telecomunicaciones.

El modelo tiene como objetivo fortalecer los proyectos de telecomunicaciones en el sector petrolero, desde su inicio y hasta el final, tomando en cuenta la evaluación de la planificación, organización y ejecución, desde el punto de vista técnico - económico, para así, tomar las medidas necesarias a través de una serie de acciones definidas de manera específica.

\section{Fases de la propuesta}

El modelo se basa en los criterios establecidos en la PMBOK (2017), Cartay (2010), Pérez (2002) y Aramburú (2001), para lograr la evaluación de proyectos de telecomunicaciones en la industria petrolera, siguiendo así mismo, las indicaciones de la GGPIC (PDVSA, 1999), donde se establecen lineamientos para los proyectos en la industria petrolera, y específicamente en telecomunicaciones; así como también, su ejecución en fases temporales claramente definidas. De esta manera, las fases del modelo propuesto se dividen según el tiempo o nivel de madurez que tiene el proyecto, donde cada fase acompaña a una serie de requerimientos que deben cumplirse para pasar al siguiente nivel o fase de madurez.

La evaluación se debe realizar al proyecto durante su ciclo de vida; de esta manera, las fases del modelo de evaluación propuesto se presentan a lo largo de su ejecución, para lo cual se proponen cuatro fases, las cuales son: 1) Ex-ante, 2) continua o en proceso, 3) Ex-post y 4) de impacto. Las mismas se muestran en el flujograma 1 , que se presenta en la Figura 6.

Figura 6. Flujograma sobre la propuesta del modelo para la evaluación de proyectos de telecomunicaciones.
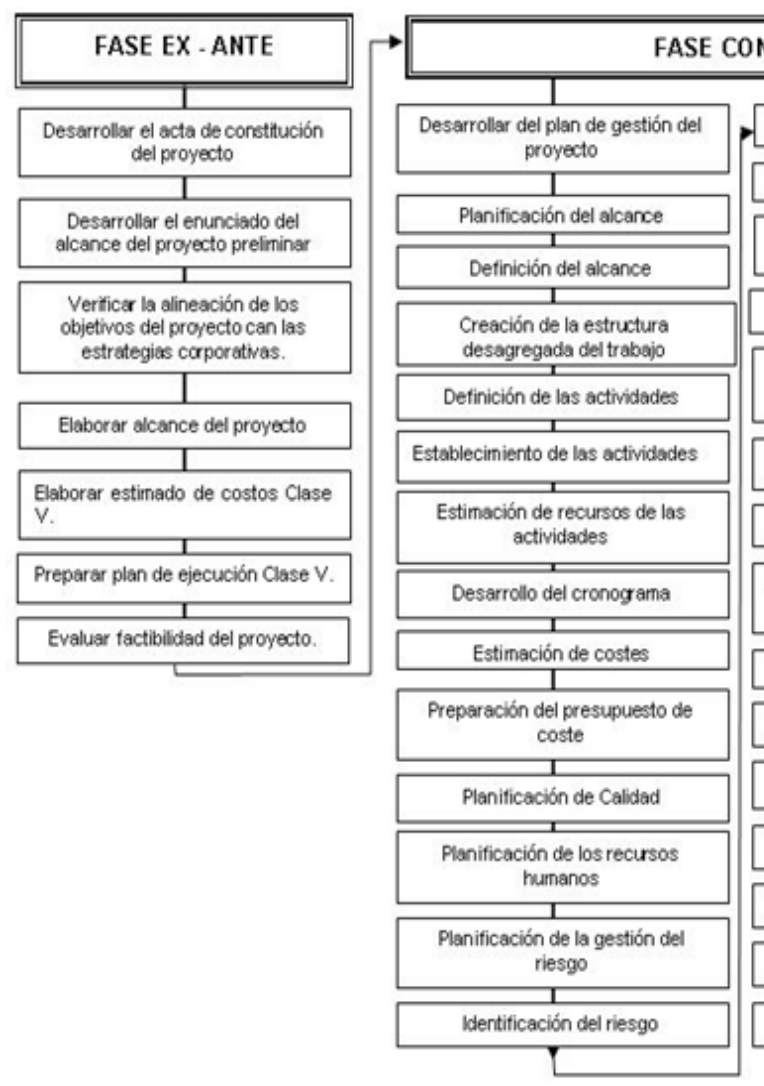

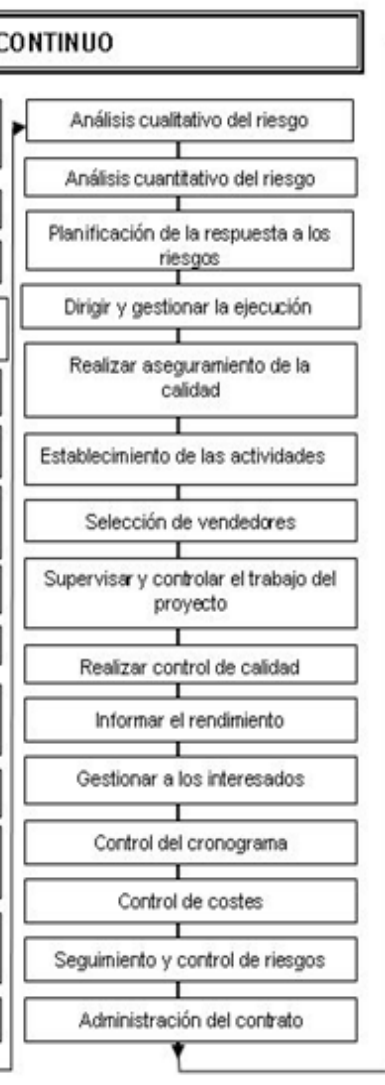

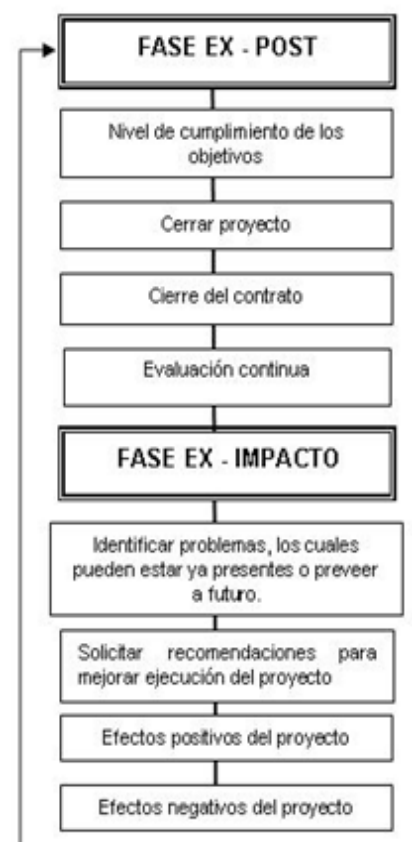
| ISNN-L: 2788 - 6549 | www revistamaya or 


\section{Fase ex - ante}

En esta fase se contempla la iniciación de un proyecto de telecomunicaciones en el sector petrolero, se inicia con un análisis, donde se determinan aquellos aspectos necesarios para la evaluación y ejecución del proyecto de telecomunicaciones, los cuales se muestran en la figura 6 correspondiente al flujograma sobre la propuesta.

La evaluación en esta fase permite conocer la factibilidad técnica, así como lo que produce el mayor impacto al mínimo costo, la fase se efectúa antes de la aprobación del proyecto y busca determinar su pertinencia, viabilidad y eficacia potencial; además, contempla todas las necesidades expuestas por el cliente. Para la ejecución de esta fase, es necesario tener claro el alcance del proyecto, elaborar un estimado clase $V$, que es el primero que se elabora y tiene un grado de precisión baja, ya que se desarrolla con información macro o burda de lo que se desea con el proyecto. De igual forma, se elabora el plan inicial de ejecución del proyecto y se determina su factibilidad técnica y económica.

Cabe resaltar que, esta fase es la ideal para realizar los ajustes que se requieran en el proyecto, los cuales se pueden incorporar en el diseño del mismo. Así mismo, se consideran las estimaciones y pronósticos financieros, de costos y beneficios; este tipo de evaluaciones permite decidir si el proyecto tiene viabilidad de materializarse o no, por ello, es recomendable se ejecuten todos los pasos que se indican en el flujograma (Figura 6).

\section{Fase en continuo}

En cuanto a la fase en continuo, en ella se contemplan las actividades y elementos para evaluar el desarrollo de un proyecto, en este caso específico, un proyecto de telecomunicaciones para el sector petrolero en Venezuela; es la fase donde se realiza un análisis, cuando se determinan aquellos aspectos necesarios para la evaluación y ejecución del proyecto.
Cabe destacar que, esta fase guarda estrecha relación con el monitoreo del proyecto. Permite conocer en qué medida se está logrando el cumplimiento de los objetivos (Resultados en caso de marco lógico); por lo que una evaluación de este tipo debe buscar aportar al perfeccionamiento del modelo de intervención empleado y a identificar lecciones aprendidas. Las fuentes financieras suelen requerir la realización de este tipo de evaluación para ejecutar los desembolsos periódicos.

La fase en continuo está conformada por veintinueve pasos, los cuales se enuncian en el flujograma (Figura 6). Es importante que los mismos se realicen, dado que conllevan a la obtención del análisis cualitativo y cuantitativo de los proyectos de telecomunicaciones, y a su vez, permiten planificar la respuesta a los riesgos, el cómo atacarlos, por lo que se hacen necesarios para decidir cómo abordar, planificar y ejecutar las actividades de gestión de riesgos para un proyecto. De igual forma en esta fase se desarrollan opciones y acciones para mejorar las oportunidades y reducir las amenazas a los objetivos del proyecto.

Una parte bien importante en esta fase es que permite gestionar el contrato y la relación entre el comprador y el suplidor, revisar y documentar, aunado a que se logra determinar el rendimiento de un suplidor o proveedor, además de gestionar la relación contractual con el comprador externo del proyecto.

\section{Fase ex - post}

La fase ex - post se realiza cuando culmina el proyecto. Esta se enfoca en indagar el nivel de cumplimiento de los objetivos (Propósito y Resultados en el caso del marco lógico): Asimismo, busca demostrar que los cambios producidos son consecuencia de las actividades del proyecto (exclusivamente o en interacción con otras fuentes) y para esto suele recurrir a un diseño experimental. 
No solo indaga por cambios positivos, también analiza efectos negativos e inesperados.

De igual forma, en esta fase se cierra formalmente el proyecto o una fase del mismo. Se tiene a disposición de la unidad de negocios o dueño de la inversión, la información necesaria para establecer una adecuada contabilidad de los activos fijos del proyecto de telecomunicaciones. Esto se realiza de acuerdo con las normas y procedimientos establecidos para tal fin por la filial y para cumplir con los requerimientos de la gerencia de finanzas, en cuanto a la capitalización de los activos nuevos o remodelados. Para ello, se siguen los pasos presentados en el flujograma (Figura 6).

El objetivo en esta fase es realizar el correspondiente recobro de la inversión y evitar cargos contables al proyecto luego de ser completado. El cierre del proyecto debe efectuarse en un lapso no mayor de tres meses, luego de la puesta en funcionamiento de las instalaciones, para poder observar en este tiempo cualquier detalle pendiente del mismo.

\section{Fase ex - impacto}

Es importante que, al finalizar el proyecto en el área de telecomunicaciones, se sostenga una reunión entre la gerencia de gestión de necesidades y oportunidades e implantación de soluciones, juntamente con la de telecomunicaciones, a fin de evaluar el cumplimiento de los objetivos, verificar los costos reales del proyecto y registrar el tiempo en que se terminó el mismo.

De igual manera, en esa reunión se deben identificar al mismo tiempo, las razones del incumplimiento de alguna de las variables, con la finalidad de encontrar insumos que, al momento de evaluar los riesgos y las oportunidades, ayuden a un mejor desarrollo de proyectos futuros. Entre los tópicos a considerar en esas reuniones podrían estar: cómo lograr metas y objetivos comunes, revisión del proyecto para determinar el estatus del mismo, identificar problemas presentes o prever inconvenientes, pedir recomendaciones para mejorar la ejecución del proyecto, realizar seguimiento al cumplimiento de asignaciones, comunicarse con todos los miembros del equipo para educar y motivar al personal del proyecto, así como conocer sobre los efectos positivos y negativos del proyecto, entre otros.

Todas las fases expuestas para la evaluación de proyectos de telecomunicaciones en la petrolera estatal se pueden aplicar, y son factibles en cualquier área de la organización que desarrollen proyectos, en virtud de la posibilidad que tiene la empresa al contar con los recursos (financieros, humanos y técnicos, entre otros) requeridos para establecer este modelo y lograr el éxito de este.

\section{CONCLUSIONES}

Entre las consideraciones finales derivadas de los objetivos planteados y de los resultados obtenidos al aplicar el instrumento de recolección de datos, se encuentran las siguientes:

La organización cuenta con personal que posee conocimiento para la realización del proceso de evaluación, sin embargo, existen oportunidades de mejora en términos de fortalecer la forma de aplicación de los aspectos que se manejan en la evaluación de proyectos.

Se determinó la aplicación de las etapas de los proyectos como visualización, conceptualización, definición, implantación y operación, asimismo se comprende el manejo de los criterios principales establecidos en las diferentes etapas señaladas, lo cual es propicio para la implantación del diseño propuesto, y superar así las debilidades presentadas en la aplicación de los aspectos referidos a la evaluación.

Se evidenció la necesidad de establecer, en la industria zona occidente del país, programas de actualización permanente sobre las tecnologías de telecomunicaciones, así como del proceso de evaluación de proyectos para coadyuvar a solventar las contingencias. 
Se sugiere la aplicación del modelo propuesto, en virtud del conocimiento de actividades y procesos inmersos en las diferentes etapas que integran el referido modelo de evaluación de proyectos, y para aprovechar al mismo tiempo, la formación que en esa materia tiene gran parte del personal del área de telecomunicaciones.

\section{REFERENCIAS}

Aramburú, C (2001). Diagnóstico, línea basal y población objetivo, Gerencia social. Diseño, monitoreo y evaluación de proyectos sociales. Universidad del Pacífico.Lima-Perú

Banco Interamericano de Desarrollo (BID) (1997). Evaluación: Una herramienta de gestión para mejorar el desempeño de los proyectos. New York, USA: BID

Cartay, I. (2010) Gestión de Proyectos. Un enfoque PDVSA. Editorial Torococo. Maracaibo. Edo Zulia. Venezuela

Crosby, W. (1998). Proceso. México. Mc Graw Hill Editores

David, F. (2013). Conceptos de administración estratégica. Desima 4ta Edición. Editorial Pearson. México

Gido, J. y Clements, J. (1999). Administración exitosa de proyectos. 3ra edición Cengage learning

Hewett, T. (1986). The role of iterative evaluation in designing systems usabitity. Proceeding of the 2nd. BCS HCl

Homs, C. (2003). La tecnología de la información: herramienta esencial para gestionar la productividad. En: VII Jornada EUROCOM2BDigital: Internet como una herramienta de negocio en la empresa. Barcelona
Medianero, B. (1998). Sistema de diseño de proyectos de cooperación técnica. CEMPRO. Lima, Perú

Pérez, J. (2002), Estudios de modelos de evaluaciones de proyectos de inversión en $\mathrm{Tl} /$ SI. DIICC. Universidad de Concepción

PDVSA (1999), Guía de gerencia para inversión capital (GGIPC). Comité de operaciones de PDVSA. Venezuela

Project Management Institute (PMBOK) (2017). Guía de los Fundamentos de la Dirección de Proyectos. 6ta. Edición. Newtown Square, Pennsylvania

Remeyi, D., Money, A., Sherwood-Smith, M., e Irani (2000). Why evaluation information tecnology investments. In Butterworth \& Heinemann (Eds). The effective measurement of management of IT costs and benefits. 2da edición. MA: Butterworth Heinemann

Ruíz, B. (2002). Instrumentos de investigación educativa. Procedimiento para su diseño y validación. CIPEG, Barquisimeto

Sapag, N. (2014); Preparación y evaluación de proyectos. 5ta edición. México, Mc Graw Hill

Strassmann, P. (1997). The Squandered Computer. Evaluating the bussiness Alignment of information technologies. Ed. The informaion Economics Press. USA 